\title{
Genetic analysis of 17 Y-STR loci in Han and Korea populations from Jilin
}

\section{Province, Northeast China}

Yinan $\mathrm{Han}^{1,2 \dagger}$, Liming $\mathrm{Li}^{2 \dagger}$, Xiaoyang $\mathrm{Liu}^{3}$, Wenqing Chen ${ }^{4}$, Shuping Yang ${ }^{2}$, Lanhai Wei ${ }^{2}$, Mingying $\mathrm{Xia}^{2}$, Teng $\mathrm{Ma}^{2}, \mathrm{Li} \mathrm{Jin}^{2 *}$, Shilin $\mathrm{Li}^{2^{*}}$

${ }^{1}$ Department of ophthalmology, Eye and Ent hospital of Fudan University, Shanghai, China

${ }^{2}$ Ministry of Education Key Laboratory of Contemporary Anthropology and State Key Laboratory of Genetic Engineering, Collaborative Innovation Center for Genetics and Development, School of Life Sciences, Fudan University, Shanghai, China

${ }^{3}$ China-Japan Union Hospital, School of Pharmaceutical Sciences, Jilin University, Changchun, China

${ }^{4}$ Jilin Province Cancer Hospital, Changchun, China

${ }^{\dagger}$ The two authors contributed equally to the work.

\section{*Corresponding author:}

Dr. Li Jin, School of Life Sciences, Fudan University, 2005 Songhu Road, Shanghai 200438, China

Tel: 8602151630607

Fax: 8602151630607

Email: lijin@fudan.edu.cn

Dr. Shilin Li, School of Life Sciences, Fudan University, 
2005 Songhu Road, Shanghai 200438, China

Tel: 8602151630602

Fax: 8602151630607

Email: lishilin@ fudan.edu.cn 
1 Genetic analysis of 17 Y-STR loci in Han and Korean

2 populations from Jilin Province, Northeast China

3

4

5 In this study, $17 \mathrm{Y}$ chromosomal short tandem repeats (Y-STRs) were analyzed in 302

6 male individuals from the Chinese Han and Korean populations of Jilin Province. The

7 haplotype diversities of two populations reached 0.99969 and 0.99874 , respectively.

8 The Jilin Han and Korean populations differed from each other significantly. The Jilin

9 Han population showed no significant difference from almost any other Han population, but it did show significant differences from most other Chinese ethnic populations. The haplotype frequencies in the Jilin Korean population studied here $\underline{\text { showed significant differences from all reference populations in earlier reports. These }}$ data provide a reference for the Y-STR database in Jilin Province, and they may be $\underline{\text { valuable for population genetic analysis. }}$

\section{Introduction}

Jilin Province is located in northeastern China (Fig. S1). According to the 2000 census, at which point the population of Jilin had reached to $26,802,191$ and contained 44 ethnic groups. Among them, Han were the most numerous $(90.85 \%)$, followed by Chinese Koreans (4.27\%), Manchus (3.71\%), Mongolians (0.64\%), and Hui (0.47\%). The composition of Jilin population has been profoundly changed by population migrations. In particular, the Qing government began to open this area in 1860 , 
enabling a large number of Han Chinese migrants to enter. They came primarily came from Shandong Province in eastern China. Koreans also migrated into Jilin during the $\underline{\text { same period, coming mainly from what is now northern Korea and most of them live }}$ in the Yanbian Korean Autonomous Prefecture.

\section{Materials and methods}

\subsection{Samples and experiments}

A total of 302 male individuals were collected from Jilin Province, including 196 Hans (Jilin, China [Han]) and 106 Koreans (Jilin, China [Korean]). All individuals provided informed consent, and then peripheral blood samples were collected. Genomic DNA was extracted using Gentra Puregene Blood Kit (Qiagen). Polymerase chain reaction (PCR) was performed using the AmpFlSTR Yfiler ${ }^{\mathrm{TM}}$ PCR Amplification Kit (Thermo Fisher Scientific Company, Carlsbad, CA, U.S.) in the GeneAmp PCR System 9700 (Thermo Fisher Scientific Company) according to the manufacturer's instructions. A total of 17 Y-STRs were analyzed, including DYS19, DYS389I, DYS389II, DYS390, DYS391, DYS392, DYS393, DYS437, DYS438, DYS439, DYS448, DYS456, DYS458, DYS635, YGATAH4, and DYS385a/b. PCR products were separated by capillary electrophoresis in ABI PRISM 3130xL Genetic Analyzer (Thermo Fisher Scientific Company). The GeneMapper ID software v3.2 (Thermo Fisher Scientific Company) was used for genotype assignment. DNA typing and assignment of nomenclature was based on the ISFG recommendations [1].

\subsection{Statistical analyses}

The haplotype frequencies were estimated using direct counting. Haplotype diversity 
was calculated as described by Nei [2]. The algorithm is $\mathrm{HD}=(\mathrm{n} / \mathrm{n}-1)\left(1-\sum \mathrm{p}_{\mathrm{i}}^{2}\right)$, where $\mathrm{n}$ is the sample size and $\mathrm{p}_{\mathrm{i}}$ is the frequency of the ith haplotype. Pairwise Rst was estimated between populations of the present study and other reports involving Chinese populations using Arlequin v3.5 [3]. Results were visualized using multidimensional scaling plot (MDS) with $\mathrm{R}$ v3.1.2 (http://www.r-project.org). DYS385 a/b was excluded from the analysis, and DYS389B was obtained by subtracting the allele of DYS389I from that of DYS389II.

\section{Results and discussion}

\subsection{Haplotype distributions}

A total of 190 different haplotypes were observed among the individuals in the Han population of the present study, of which 184 were unique and 6 were shared in two individuals. A total of 100 different haplotypes were observed among Koreans, of which 95 were unique, 4 were shared by two individuals, and 1 was shared by three individuals. The haplotype diversities of Han and Korean were 0.99969 and 0.99874 , respectively.

\subsection{Genetic distance and population comparison}

Pairwise Rst was estimated between populations in the present study and other reports involving Chinese populations based on 17 Y-STR loci. Overall, the haplotype frequency data of 26 Chinese populations were obtained from earlier reports [4-21], which included 14 Han [5-14] and 12 Chinese ethnic populations [6, 14-21], and a total of 8,784 reference samples were analyzed. As shown in Table S2, Han and Korean populations in Jilin Province showed significant differences after Bonferroni 
correction $(\mathrm{p}>0.05 / 378=0.0001323)$. No significant differences were observed between Jilin Han and the other Han populations in earlier reports, except Zhejiang, China [Han] [5] and Luzhou, China [Han] [9]. The results showed that most of Han populations in the present study had substantial homogeneity based on 17 Y-STRs, and significant differences in haplotype frequencies were observed between the Han populations of and Jilin and Zhejiang and between that of Jilin and Luzhou. These differences are probably attributable to different sampling locations. Results showed $\underline{\text { that Jilin Han differed significantly from most Chinese ethnic populations. Among }}$ $\underline{\text { them, Jilin Han showed significant differences from all the Chinese ethnic populations }}$ in southern China, including Hunan [Dong] [6], Hunan [Miao] [6], Hunan [Tujia] [6], Guangxi [Zhuang] [14], and Taiwan [Paiwan] [21]. Jilin Han also showed significant differences from some Chinese ethnic populations from northern China, including Lassa [Tibetan] [17], Qinghai [Tibetan] [18], Xinjiang [Kazakh] [19], and Xinjiang [Uyghur] [19]. The results suggested that Jilin Han had population structure and history different from these populations. In addition, the haplotype frequencies of Jilin Koreans in the present study showed significant differences from all reference populations in earlier reports, indicating a distant genetic relationship between Jilin Korean and the other populations in this study. The details are shown in Table S2, and the same conclusions are given in Figure S2.

\section{Conclusions}

In conclusion, we report the haplotype frequency and forensic parameters based on 17 Y-STR loci of Han and Korean populations in Jilin Province. We believe the data is 
valuable for both forensics and population genetics.

Our data has been submitted to YHRD, and received the accession number: Jilin,

China [Han], n=196, YA004124, and Jilin, China [Korean], n=106, YA004126.

The study follows the new guidelines for publication of population data requested by the journal $[22,23]$.

\section{Acknowledgments}

This study was supported by grants from the Genographic Project, the National Science Foundation of China (31401060), National Science Foundation of China (31271338), Project of Chinese Ministry of Education (113022A) and National High Technology Research and Development Program (2012AA021802).

\section{Reference}

[1] Gusmao L, Butler JM, Carracedo A, Gill P, Kayser M, Mayr WR, Morling N, Prinz M, Roewer L, Tyler-Smith $C$ et al: DNA Commission of the International Society of Forensic Genetics (ISFG): An update of the recommendations on the use of Y-STRs in forensic analysis. Forensic Scilnt 2006, 157(2-3):187-197.

[2] Nei M: Molecular evolutionary genetics. New York: Columbia University Press; 1987, pp. 176-179.

[3] Excoffier L, Lischer HEL: Arlequin suite ver 3.5: a new series of programs to perform population genetics analyses under Linux and Windows. Mol Ecol Resour 2010, 10(3):564-567.

[4] Jing $X u$, Liming Li, Lanhai Wei, Zhiyi Nie, Shuping Yang, Mingying Xia, Teng Ma, Hui Sun, Xueying Zhao, Yuan Ping, Huaigu Zhou, Fuzhong Xue, Ziqin Zhao, Li Jin, Shilin Li: Genetic analysis of 17 Y-STR loci in Han population from Shandong Province in East China. submitted.

[5] Li Liming, Yu Ge, Li Shilin, Jin Li, Yan Shi: Genetic analysis of 17 Y-STR loci from 1,019 individuals of six Han populations in East China. submitted.

[6] Shu Lili, Li Liming, Yu Ge, Yu Binxia, Liu Yuchi, Li Shilin, Jin Li, Yan Shi: Genetic analysis of 17 Y-STR loci in Han, Dong, Miao and Tujia populations from Hunan province, central-southern China. Forensic Sci Int-Genet 2015, 07. 007. 
[7] Li Liming, Ma Teng, Xu Jing, Yang Yajun, Yang Shuping, Xia Mingying, Sun Hui, Zhao Xueying, Ping Yuan, Zhou Huaigu, Xie Jianhui, Zhao Ziqin, Jin Li, Li Shilin: Genetic analysis of 17 Y-STR loci in Han population from Gansu Province in northwestern China. Forensic Sci Int-Genet 2015, 19:134-135.

[8] Yang YY, Yuan WL, Guo F, Jiang XH: Population data of 17 Y-STR loci in Nanyang Han population from Henan Province, Central China. Forensic Sci Int-Genet 2014, 13:145-146.

[9] Bing L, Liang WB, Pi JH, Zhang DM, Yong D, Luo HB, Zhang LS, Lin Z: Population genetics for 17 Y-STR loci(AmpFISTR (R) Y-filerTM) in Luzhou Han ethnic group. Forensic Sci Int-Genet 2013, 7(2):E23-E26.

[10] Liu Y, Liao LC, Gu MB, Ye Y: Population genetics for 17 Y-STR loci in a Chinese Han population sample from Mudanjiang city, Northeast China. Forensic Sci Int-Genet 2014, 13:E16-E17.

[11] Bai RF, Zhang Z, Liang QZ, Lu D, Yuan L, Yang X, Shi MS: Haplotype diversity of 17 Y-STR loci in a Chinese Han population sample from Shanxi Province, Northern China. Forensic Sci Int-Genet 2013, 7(1):214-216.

[12] Shi Meisen, Liu Yaju, Zhang Juntao, Bai Rufeng, Lv Xiaojiao, Ma Shuhua: Analysis of 24 Y chromosomal STR haplotypes in a Chinese Han population sample from Henan Province, Central China. Forensic Sci Int-Genet 2015, 17:83-86.

[13] Wang Dan, Liu Faqin, Kong Lingxue, Yuan Zhenyu, Chen Guang, Ye Jian: Population data of 17 Y-STR haplotypes in Jining Han population from Shandong province, East China. Forensic Sci Int-Genet 2015.05.017.

[14] Luo Haibo, Song Feng, Zhang Lushun, Hou Yiping: Genetic polymorphism of 23 Y-STR loci in the Zhuang minority population in Guangxi of China. Int J Legal Med 2015, 129(4):737-738.

[15] Guo F: Population genetics for 17 Y-STR loci in Mongolian ethnic minority from Liaoning Province, Northeast China. Forensic Sci Int-Genet 2015, 17:153-154.

[16] Guo F, Zhang L, Jiang XH: Population genetics of 17 Y-STR loci in Xibe ethnic minority from Liaoning Province, Northeast China. Forensic Sci Int-Genet 2015, 16:86-87.

[17] Li KL, Kai L, Yuming M: Y chromosome STR haplotypes of Tibetan living Tibet lassa. Forensic Scilnt 2007, 172(1):79-83.

[18] Zhu BF, Wu YM, Shen CM, Yang TH, Deng YJ, Xun X, Tian YF, Yan JC, Li T: Genetic analysis of 17 Y-chromosomal STRs haplotypes of Chinese Tibetan ethnic group residing in Qinghai province of China. Forensic Scilnt 2008, 175(2-3):238-243.

[19] Shan WJ, Ablimit A, Zhou WJ, Zhang FC, Ma ZH, Zheng XF: Genetic polymorphism of 17 Y chromosomal STRs in Kazakh and Uighur populations from Xinjiang, China. Int J Legal Med 2014, 128(5):743-744.

[20] He J, Guo F: Population genetics of 17 Y-STR loci in Chinese Manchu population from Liaoning Province, Northeast China. Forensic Sci Int-Genet 2013, 7(3):E84-E85.

[21] Wu FC, Ho CW, Pu CE, Hu KY, Willuweit S, Roewer L, Liu DH: Y-chromosomal STRs haplotypes in the Taiwanese Paiwan population. Int J Legal Med 2011, 125(1):39-43.

[22] Carracedo A, Butler JM, Gusmao L, Linacre A, Parson W, Roewer L, Schneider PM: New guidelines for the publication of genetic population data. Forensic Sci Int-Genet 2013, 7(2):217-220.

[23] Carracedo A, Butler JM, Gusmao L, Linacre A, Parson W, Roewer L, Schneider PM: Update of the guidelines for the publication of genetic population data. Forensic Sci Int-Genet 2014, 
10:A1-A2.

165 